\title{
Construction of real-time monitoring system for Grid services based on log analysis at the Tokyo Tier-2 center
}

\author{
T. Kishimoto*1, T. Mashimo ${ }^{1}$, N. Matsui ${ }^{1}$, T. Nakamura ${ }^{2}$, H. Sakamoto ${ }^{1}$ \\ ${ }^{1}$ International Center for Elementary Particle Physics, The University of Tokyo \\ ${ }^{2}$ Computing Research Center, KEK \\ E-mail: tomoedicepp.s.u-tokyo.ac.jp
}

The Tokyo Tier- 2 center, which is located in the International Center for Elementary Particle Physics at the University of Tokyo, is providing computer resources for the ATLAS experiment in the Worldwide LHC Computing Grid. Logs produced by the Grid services provide useful information to determine whether the services are working properly. Therefore, a new real-time monitoring system based on log analysis has been constructed using the ELK stack. This paper reports the configuration of the new monitoring system at the Tokyo Tier- 2 center, and discusses improvements in terms of stability and flexibility of the site operation by introducing the new monitoring system.

International Symposium on Grids and Clouds (ISGC) 2018 in conjunction with Frontiers in Computational Drug Discovery

16-23 March 2018

Academia Sinica, Taipei, Taiwan

* Speaker. 


\section{Introduction}

The Tokyo Tier-2 center, which is located in the International Center for Elementary Particle Physics (ICEPP) [四] at the University of Tokyo, is providing computer resources for the ATLAS experiment [ []] in the Worldwide LHC Computing Grid (WLCG) [3]. The official site operation in the WLCG was launched in 2007 after several years of development. The site has been achieving a stable and reliable operation since then.

The Grid middleware is a software stack, which provides services for users to access the distributed computing resources of the Grid. A stable operation of the Grid services is a key role of the sites. Logs produced by the Grid services provide useful information to determine whether the services are working properly. Therefore, a new real-time monitoring system based on log analysis has been constructed. This paper reports the configuration of the new monitoring system, and discusses improvements in terms of stability and flexibility of the site operation by introducing the new monitoring system. The paper is organized as follows, Section 2 provides the configuration and status of the Tokyo Tier- 2 center. Section 3 describes the construction of the new monitoring system. Section 4 gives a summary.

\section{Tokyo Tier2 center}

In the Tokyo Tier- 2 center, almost all hardware devices are leased, and are replaced in every three years in order to satisfy the requirement of the ATLAS experiment. The latest hardware upgrade was done in January 2016, and the new system (so-called 4th system) is stably running. In the 4th system, 6144 CPU cores (256 worker nodes) and 7392 TB disk storages are reserved for the ATLAS experiment. Table $\square$ summarizes the WLCG pledge and actual deployed resources. It can be confirmed that the WLCG pledges are well satisfied with the 4th system. The new system will be provided for year 2019 .

\begin{tabular}{ccccc}
\hline & & CPU [HS06] & Disk [TB] & LOCALGROUPDISK [TB] \\
\hline \hline \multirow{2}{*}{2017} & Pledge & 34,000 & 4,000 & - \\
& Deployed & $111,268(6144$ CPU cores $)$ & 4,000 & 1,000 \\
\hline \multirow{2}{*}{2018} & Pledge & 40,000 & 4,800 & - \\
& Deployed & $111,268(6144$ CPU cores $)$ & 4,800 & 1,000 \\
\hline
\end{tabular}

Table 1: The WLCG pledge and deployed resources at the Tokyo Tier2 center. All deployed resources are reserved for the ATLAS experiment. LOCALGROUPDISK is dedicated disks for the ATLAS-Japan group.

\subsection{Status in ATLAS experiment}

Figure $\square$ shows the slot occupancy at the Tokyo Tier-2 center (top) and the proportion of number of completed jobs of Tier-2 sites in the ATLAS experiment (bottom). The figures show the results for year 2017. It can be seen that the slots were well filled by various types of jobs. The Tokyo Tier-2 site completed $5.5 \%$ of the jobs in Tier-2s. Also a high site availability, which is greater than $99 \%$, has been achieved using the 4 th system. 


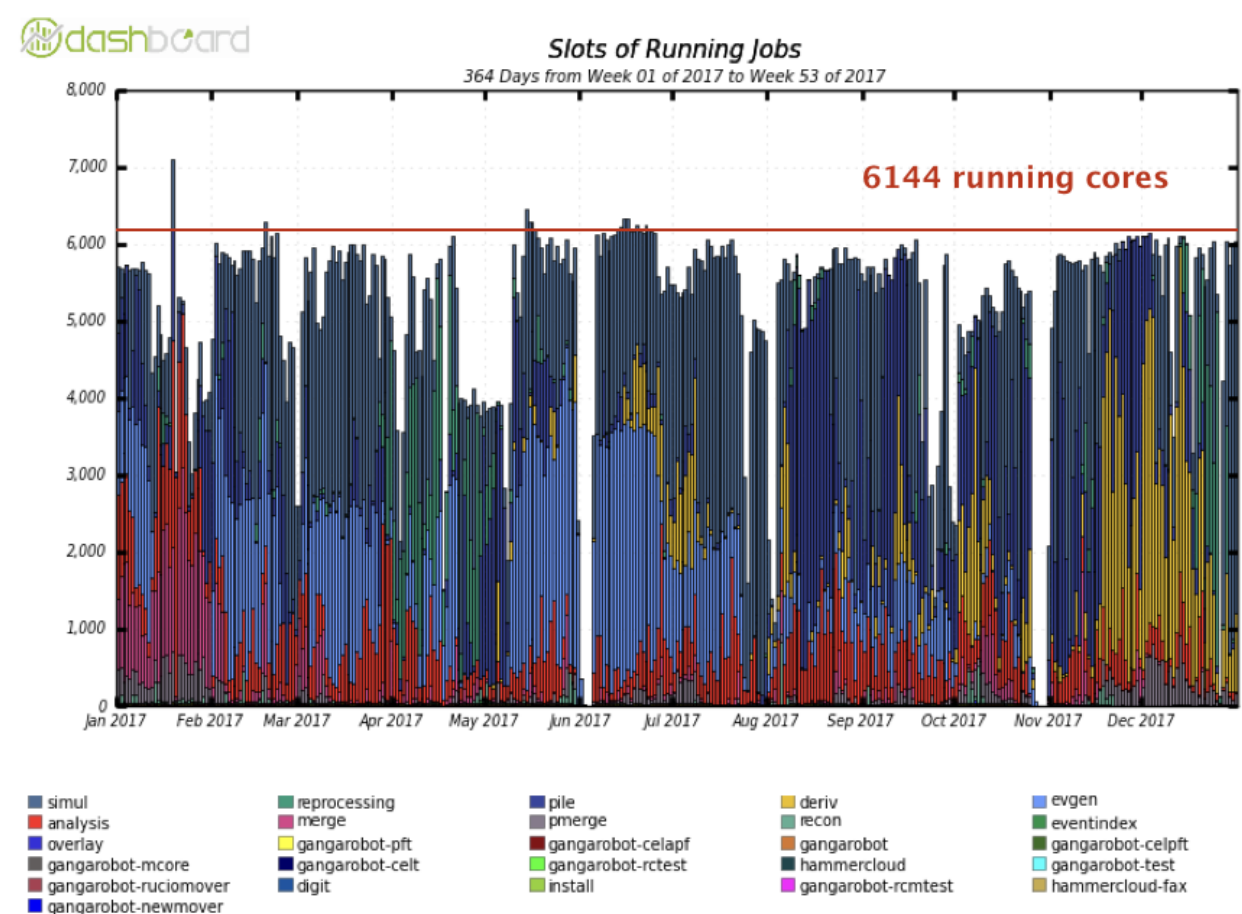

Maximum: 7,111 , Minimum: 0.00, Average: 5,121, Current: 6,041

\section{霡dashboard}

Completed jobs (Sum: 250,523,467)

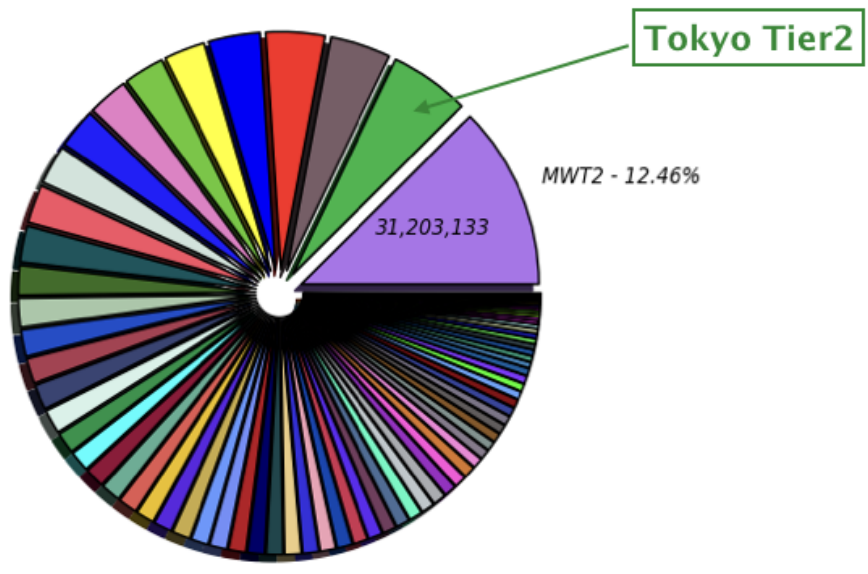

MWT2 - $12.46 \%(31,203,133)$

- SWT2 CPB - $4.12 \%(10,313,957)$

DESY-HH - $3.51 \%(8,782,936)$

UKI-LT2-QMUL - $2.75 \%(6,881,624)$

UKI-SCOTGRID-GLASGOW - $2.70 \%(6,770,520)$

UKI-NORTHGRID-MAN-HEP - $2.61 \%(6,539,7,3)$

CA.VICTORIA-WESTGRID-T2 - 2.00\% $(5,014,740)$

TRZ-LMU $1.79 \%(4,482,163)$

WUPPERTALPROD $-1.70 \%(4,250,916)$

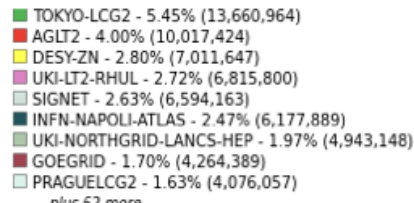

Figure 1: The slot occupancy at the Tokyo Tier-2 center (top) and the proportion of number of completed jobs of Tier-2 site in the ATLAS experiment (bottom). 


\subsection{Grid middlewares}

The ARC-CE [四] is deployed as the computing element in front of the HTCondor batch job scheduler [ []]. The configuration of the computing element is shown in Figure $\square$. Two ARC-CEs are installed for redundancy. The high availability configuration of the central manager of the HTCondor is also implemented.

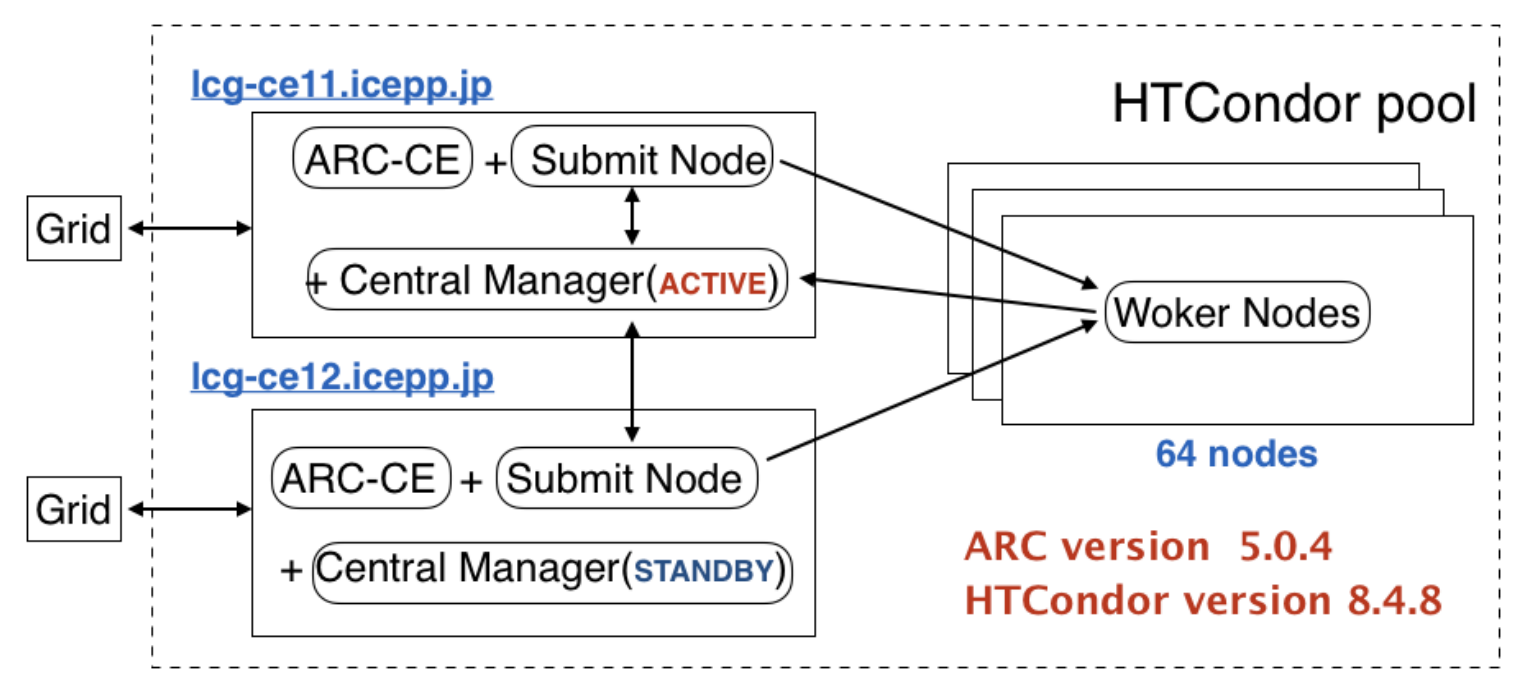

Figure 2: The configuration of the computing element at the Tokyo Tier-2 center.

The disk storage consists of 48 sets of a disk array and a file server, which are managed by the Disk Pool Manager (DPM) [焑]. The configuration of the storage element is shown in Figure [3]. The DPM supports several protocols for data transfers, such as GridFTP [ [ $]$ ], XRootD [8] and https. The MySQL [Q] is used as the database of the DPM, and the auto replication of the database is available in master-slave configuration.

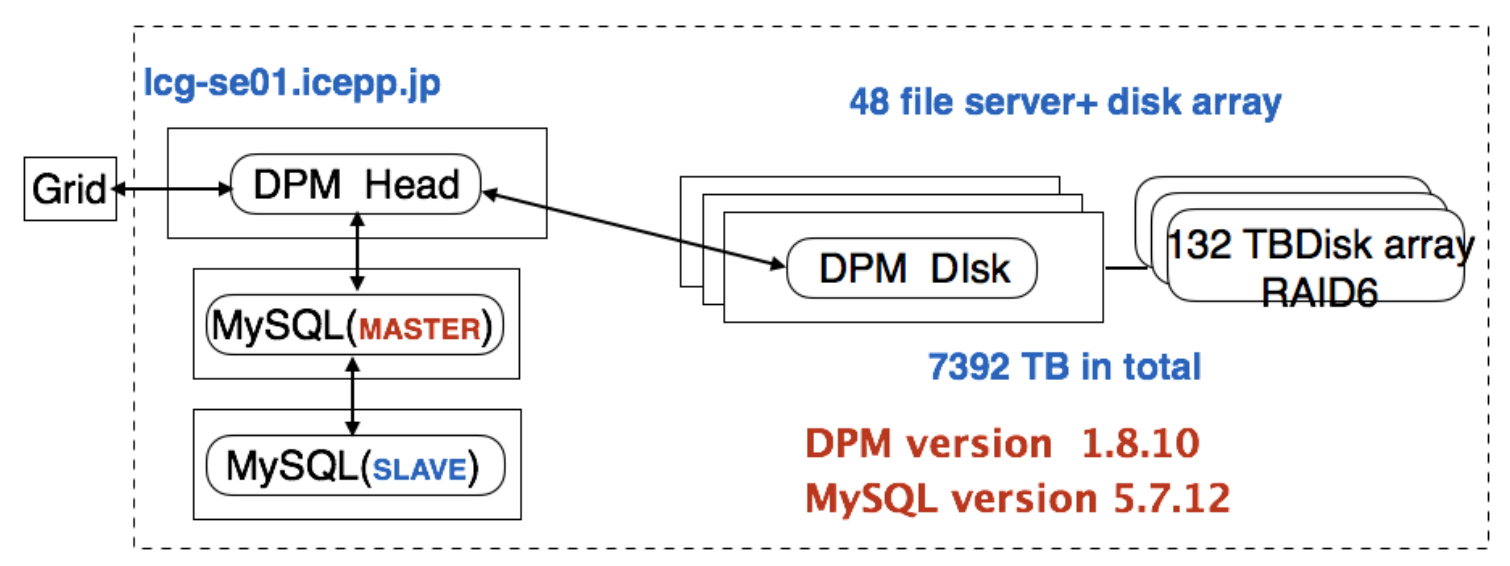

Figure 3: The configuration of the storage element at the Tokyo Tier-2 center.

\section{New monitoring system for Grid services}

Logs produced by the Grid services provide useful information to check health of the services. 
Figure $\mathbb{G}$ shows an example of text logs produced by the ARC-CE. Time stamp, severity of message, job global ID and status of job transition are shown in this example. By checking the log, we can judge that the job was processed at the ARC-CE without errors.

Time stamp Severity

2018-02-05 04:05:23] [Arc] [INF0] [6734/2] roaNDm7Cn2 rnG457 in 1NHNUqABFKDmABFKDmVi1XDmABFKDmpGQQXn: State: ACCEPTED: parsing job description

[2018-02-05 04:05:23] [Arc] [INF0] [6734/2] roaNDm7Cn2 rnG457 in1NHNUqABFKDmABFKDmVi1XDmABFKDmpGQQXn: State: ACCEPTED: moving to PREPARING

[2018-02-05 04:05:23] [Arc] [INF0] [6734/2] roaNDm7Cn2 rnG457 in1NHNUqABFKDmABFKDmVi1XDmABFKDmpGQQXn: State: PREPARING from ACCEPTED
[2018-02-05 04:05:31] [Arc.DataStaging.DTR] [INF0] [6734/7] roaNDm7Cn2 rnG457 in1NHNUqABFKDmABFKDmVi1XDmABFKDmpGQQXn: All downloads finished

successfulty

[2018-02-05 04:05:31] [Arc] [INF0] [6734/2] roaNDm7Cn2rnG457in1NHNUgABFKDmABFKDmVi1XDmABFKDmpGO0Xn: State: SUBMIT from PREPARING

[2018-02-05 04:05:31] [Arc] [INF0] [6734/2] roaNDm7Cn2rnG457in1NHNUqABFKDmABFKDmVi1XDmABFKDmpGQQXn: state SUBMIT: starting child: /usr/share/

arc/submit-condor - job

[ [

:05:32] [Arc] [INF0] [6734/2] roaNDm7Cn2rnG457 in1NHNUqABFKDmABFKDmVi1XDmABFKDmpGQQXn: State: INLRMS from SUBMIT

[2018-02-65 04:22:01] [Arc] [INF0] [6734/2] roaNDm7Cn2 rnG457in1NHNUqABFKDmABFKDmVi1XDmABFKDmpGQQXn: Job finished

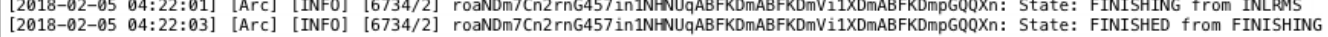

Figure 4: An example of text logs produced by the ARC-CE.

Therefore, a new real-time monitoring system based on log analysis has been constructed using the ELK stack [ए]] in order to detect a problem of the Grid services. The ELK stack consists of Elasticseach, Logstash, Kibana and Beat, and provides an efficient way of log processing, storing, query and visualization. The function of each component of the ELK stack is summarized in Table ص. Logs produced by the ARC-CE, HTCondor and DPM are processed and monitored in the new monitoring system. A dedicated Logstash configuration for each service has been developed to parse the Grid service logs. Generally, 1 line in the log file is processed and stored as 1 record in the Elasticsearch database.

\begin{tabular}{cc}
\hline Beat 5.6 & data shipper \\
Logstash 5.6 & $\log$ processing, transformation \\
Elasticsearch 5.6 & data storing, search \\
Kibana 5.6 & visualization \\
\hline
\end{tabular}

Table 2: The function of each component of the ELK stack.

\subsection{Resource usage of ELK host}

The Elasticseach, Logstash and Kibana are installed to a physical machine, which has $24 \mathrm{CPU}$ cores and 32GB memory (Dell Power Edge M630). The total capacity of local disks is 1 TB. The $\operatorname{logs}$ are collected from the service hosts to the ELK host using rsync command. Table B shows the $\log$ frequency (number of $\log$ lines) of the services. The DPM is the dominant source of logs to be processed and stored. Figure 5 shows the resource usage of the ELK host. It can be confirmed that an enough computing resource is available in the ELK host since the observed average CPU usage is stil $\sim 200 \%$ (full usage of $24 \mathrm{CPU}$ cores $=2400 \%$ ). 12 GB memory is assigned to the JVM heap of the Elasticsearch and Logstash. The size of the Elasticsearch database increases by $\sim 20 \mathrm{~GB}$ per day. Therefore, old records, which passed 1 month, are deleted from the database.

\subsection{Examples of the new monitoring}

Figure 6 shows selected figures in the new monitoring system for the ARC-CE. The left figure shows the number of job states, such as SUBMIT, ACCEPTED, PREPARING, at the computing 


\begin{tabular}{ccc}
\hline ARC-CE & HTCondor & DPM \\
\hline \hline$\sim 300 / \mathrm{min}$ & $\sim 5,000 / \mathrm{min}$ & $\sim 50,000 / \mathrm{min}$ \\
\hline
\end{tabular}

Table 3: The log frequency (number of log lines) of the ARC-CE, HTCondor, and DPM.

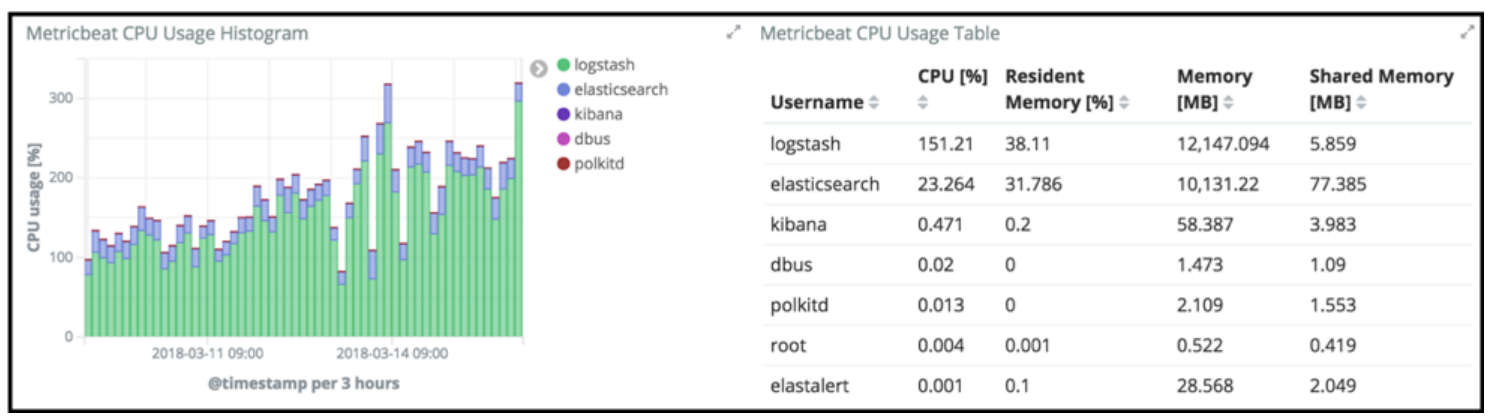

Figure 5: The resource usage of the ELK host, measured by the Metricbeat [ए]].

element. The stacked job state due to some problems in the computing element can be detected from this figure. The right figure shows the number of logs for each message severity. It can be seen that the error and warning counts are small in the figure.

Figure $\square$ also shows selected figures for the HTCondor. The left figure shows the number of completed jobs at the HTCondor. There is a good correlation between the number of FINISHED state at the ARC-CE and the number of completed jobs at the HTCondor. The right figure also shows the number of completed jobs in terms of the time it takes to complete. There is an upper limit of the job time in the HTCondor in order to kill runaway jobs. It can be monitored from this figure that the jobs are completed within the time limit.

Figure 8 shows the number of errors (left) and warning (right) for each protocol for the DPM. A bump of the warning counts can be observed in the right figure. There was a hardware problem in this period, and file deletions were failing using the httpd protocol. This experience shows a good example that the new monitoring system works well to detect a problem of the services. An alerting function is implemented using elastalert [12], which is a third party software. E-mail is sent to administrators when the error or warning counts exceed a threshold.

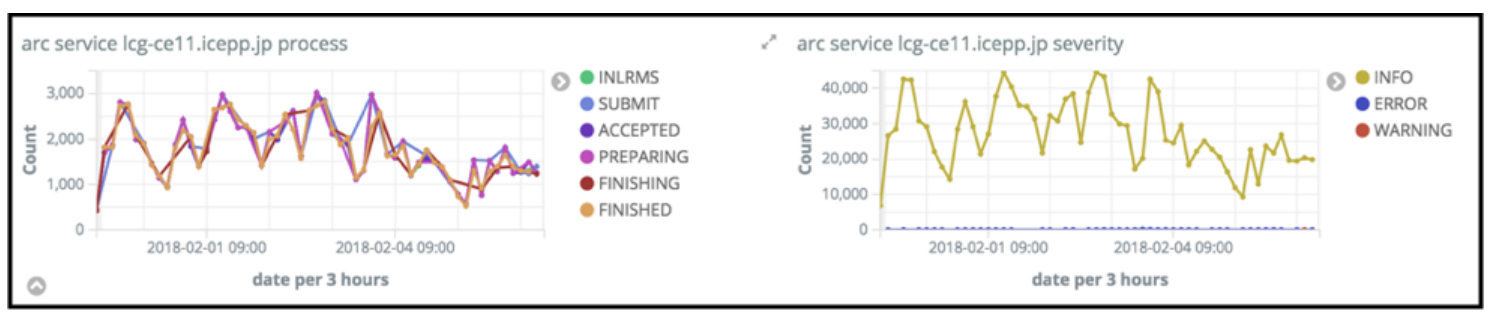

Figure 6: Examples of the ARC-CE monitoring.

\subsection{Development of iftopbeat}

The DPM supports several protocols for the data transfer. In order to measure and monitor the 


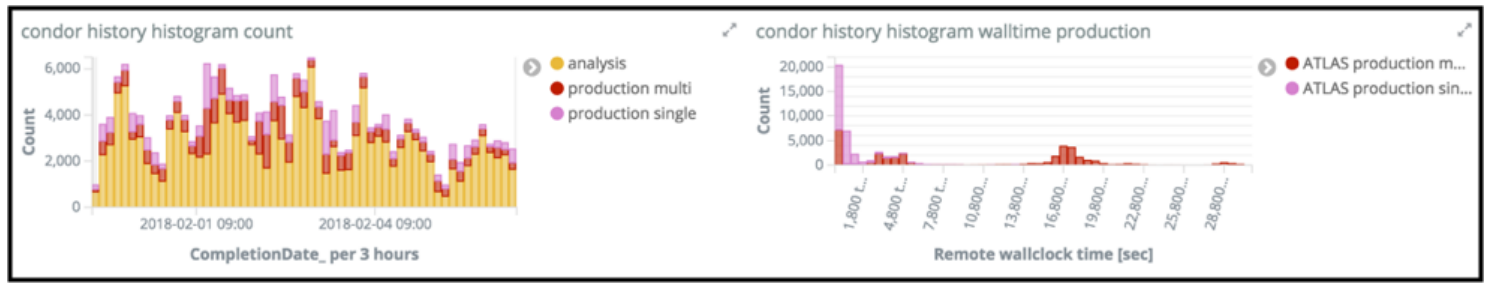

Figure 7: Examples of the HTCondor monitoring.

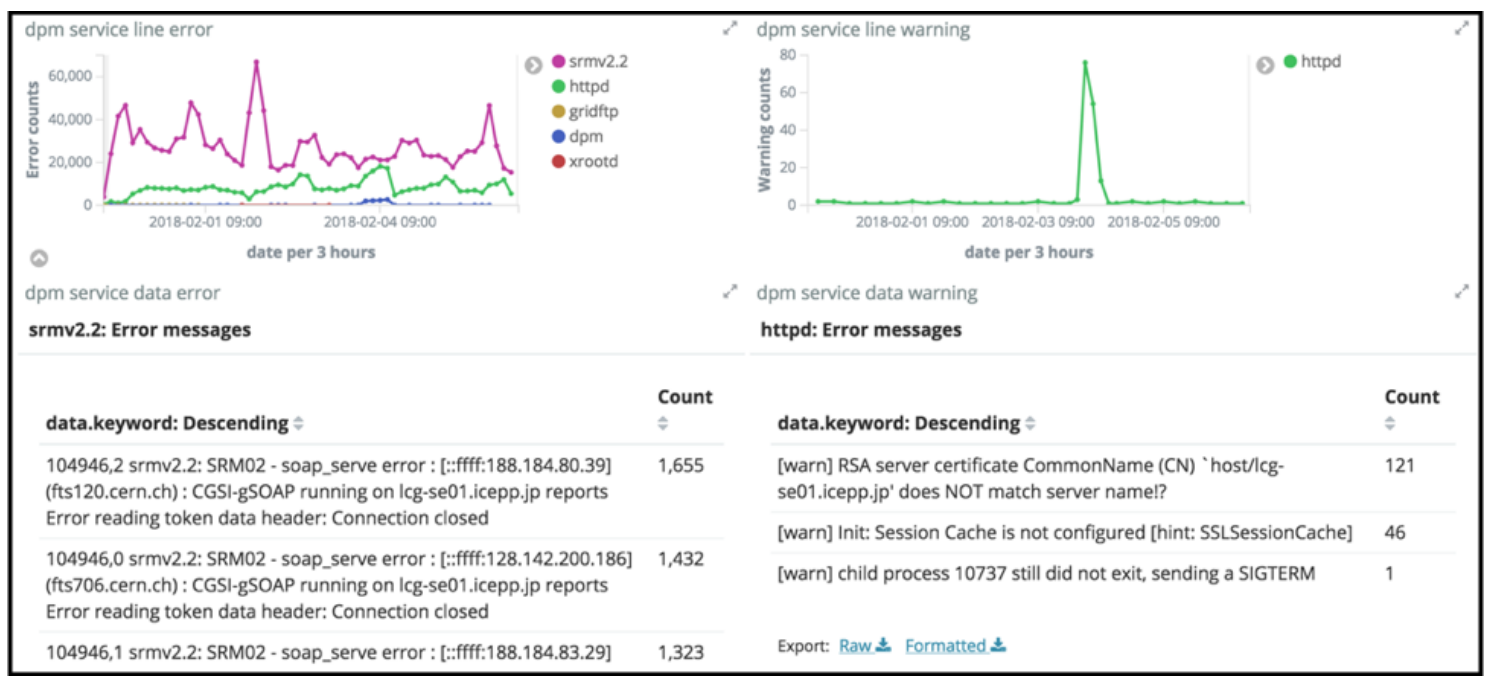

Figure 8: Examples of the DPM monitoring.

bandwidth usage of each protocol, a software (so-called iftopbeat) has been developed. Iftopbeat collects the information using iftop command, which provides the bandwidth usage, the direction and source of transfers and its ports. Then iftopbeat processes the information and directly sends the data to the Elasticsearch using the Beat libraries. Figure 8 shows the bandwidth usage of the file servers in the production system measured by iftopbeat. The protocol and transfer direction/source are well identified and visualized.

\section{Summary}

The Tokyo Tier- 2 center is providing sufficient computing resources to the ATLAS experiment in the WLCG, and is keeping the high site availability. In order to operate the site more stably and flexibly, the new real-time monitoring system based log analysis for the Grid services has been constructed using the ELK stack. It has been confirmed that the ELK stack can handle the logs produced by the Grid services at the Tokyo Tier- 2 center, and can visualize the logs well. Since the logs of the ARC-CE, HTCondor and DPM are integrated into the new monitoring system, it is easy to check a correlation between the services when a problem arises. Iftopbeat has been developed in order to monitor the bandwidth usage of each protocol, and is working well in the production system. The monitoring of the bandwidth usage for each protocol and direction/source will help to separate a problem into small parts. 


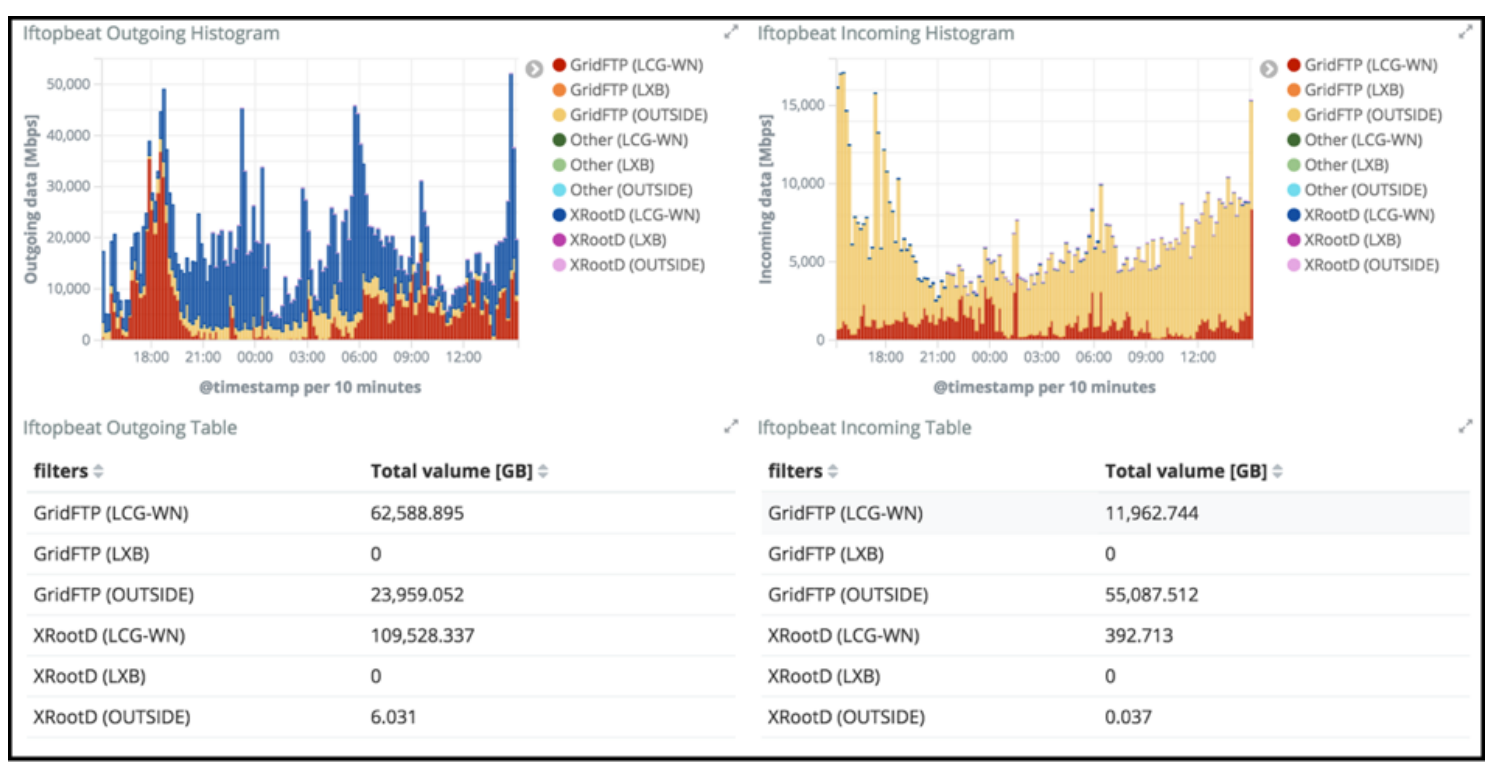

Figure 9: The bandwidth usage of the file servers in the production system, measured by iftopbeat. The left (right) figure shows outgoing (incoming) data.

\section{References}

[1] ICEPP web page, https://www.icepp.s.u-tokyo.ac.jp/en/index.html

[2] ATLAS experiment web page, http://atlas.cern/

[3] WLCG web page, http://wlcg.web.cern.ch/

[4] ARC-CE web page, http://www.nordugrid.org/arc/ce/

[5] HTCondor web page, https://research.cs.wisc.edu/htcondor/

[6] DPM web page, http://lcgdm.web.cern.ch/dpm

[7] GridFTP web page, http://toolkit.globus.org/toolkit/docs/latest-stable/gridftp/

[8] XRootD web page, http://xrootd.org/

[9] MySQL web page, https://www.mysql.com/jp/

[10] ELK stack web page, https://www.elastic.co/jp/elk-stack

[11] Metricbeat web page, https://www.elastic.co/jp/products/beats/metricbeat

[12] Eelastalert github, https://github.com/Yelp/elastalert 